\title{
Current Noise in the Vicinity of the 2D Superconductor-Insulator Quantum Critical Point
}

\author{
A. G. Green, ${ }^{1}$ J. E. Moore, ${ }^{2}$ S. L. Sondhi, ${ }^{3}$ and A. Vishwanath ${ }^{2}$ \\ ${ }^{1}$ School of Physics and Astronomy, University of St. Andrews, North Haugh, St. Andrews KY16 9SS, United Kingdom \\ ${ }^{2}$ Department of Physics, University of California, LeConte Hall, Berkeley, California 94720, USA \\ ${ }^{3}$ Department of Physics, Princeton University, Princeton, New Jersey 08544, USA
}

(Received 24 May 2006; revised manuscript received 1 September 2006; published 1 December 2006)

\begin{abstract}
Systems near to quantum critical points show universal scaling in response to external probes. We consider whether this scaling is reflected in their out-of-equilibrium fluctuations. We study current noise in the metallic state at the $z=1$ quantum critical point between a superconductor and an insulator in two dimensions. Using a Boltzmann-Langevin approach within a $1 / N$ expansion, we show that the current noise obeys a universal scaling form $S_{j}=T \Phi\left[T / T_{\text {eff }}(E)\right]$, with $T_{\text {eff }} \propto \sqrt{E}$. This treatment recovers Johnson noise in thermal equilibrium and $S_{j} \propto \sqrt{E}$ at strong electric fields. The latter differs significantly from both the shot noise in conventional metals (diffusive Fermi liquids) and the free carrier result, due to strong correlations between the critical bosonic excitations. Current-noise measurements could therefore help clarify the physics of the destruction of superconductivity in thin film superconductors.
\end{abstract}

The transition from superconducting to insulating (or metallic) behavior at zero temperature has received extensive experimental and theoretical attention over the past two decades. A wide range of physical systems - from thin films of conventional superconductors to cuprate hightemperature superconductors and bosonic atoms in optical lattices - may be tuned through a continuous transition out of their superfluid state upon varying a system parameter at low temperature. These transitions may be understood as a continuous, zero-temperature, or quantum phase transition of bosonic degrees of freedom (e.g., Cooper pairs), whose localization leads to an insulating state. Many important quantities are universal at a continuous transition, i.e., independent of material parameters and computable within a continuum field theory [1]. One such quantity is conductivity. This is a dimensionless quantity in two spatial dimensions and is expected to take on a universal value $[2,3]$. Thus, a metallic state is expected to be sandwiched between the superconducting and insulating statesalthough this occurs at a point rather than over a region of the phase diagram. In contrast to the usual origin of metallic conduction from diffusive fermions, the metallic state here is composed of critical bosons. It is interesting to compare and contrast the behaviors of these two metallic states.

Although revealing, conductivity alone cannot distinguish between the diffusive fermionic and critical bosonic metals. Two recent works of Dalidovich and Philips [4] and Green and Sondhi [5] have extended the analysis of the superconductor to insulator quantum phase transition to see whether universality persists out of equilibrium. Surprisingly, it does, at least in transport. The vanishing energy scales at the critical point lead to universal nonlinear conductivity even when the system is driven away from thermal equilibrium by the application of a strong electric field. Unfortunately, for the two-dimensional su- perconductor to insulator (SI) transition, this nonlinearity in conductivity is trivial (the conductivity is linear even at high fields, although of a different magnitude to the linear response value) and does not provide a clear differentiation between the two metallic phases.

In this work, we consider another experimentally measurable quantity - current noise. We demonstrate that the high-field noise power of the critical bosonic metal shows a qualitative difference from that in an ordinary metal [6]. Hence, noise measurements allow a simple, sharp distinction between the critical point of the SI transition and an ordinary metal and can inform debates over whether metallic resistivity near the SI transition indicates a conventional metallic phase or a broadened quantum phase transition. Measurements of current fluctuations are usually restricted to mesoscopic samples in order that the relative fluctuations of current be significant; such samples have relatively few conducting channels giving a total conductance of order the conductance quantum $e^{2} / h$. Quantum critical systems may also satisfy this criterion. For example, at the two-dimensional SI transition, the conductance is also of order the conductance quantum [2-5].

Our results, obtained using a Boltzmann-Langevin equation [7,8] within a $1 / N$ expansion, are as follows: In thermal equilibrium, we recover Johnson noise with noise power $S_{j}=4 \sigma T$, in accord with the equilibrium fluctuation-dissipation relation. This crosses over at very large electric fields to $S_{j} \propto \sqrt{E}$. This is strongly suppressed from the usual shot-noise result of $S_{j} \propto j \propto E$ due to the strong correlations at the quantum critical point. Between these two limits, the current noise obeys a universal scaling form $S_{j}=T \Phi\left[T_{\text {eff }}(E) / T\right]$.

The rest of this Letter is organized as follows: We begin by briefly outlining the reasons for seeking a BoltzmannLangevin description of the current noise at the SI transition. After this, we turn to the main part of our calculation. 
We outline the field-theoretical formulation of the particlehole symmetric, bosonic, superfluid to insulator transition and how a Boltzmann equation for transport may be obtained from it. We then summarize the scaling of the resulting distribution function in thermal equilibrium and in the high-field regime. Next, we introduce the Boltzmann-Langevin equation. We show how the scaling of the distribution function leads to a current noise that crosses over from Johnson noise at low fields to an unusual, but universal, $\sqrt{E}$ dependence in the strong-field limit. Finally, we turn to a discussion of the interpretation and experimental implications of these results.

The Boltzmann-Langevin equation $[7,8]$ has been used extensively in the study of current noise in fermionic systems. This "kinetic theory of fluctuations" approach gives the correct leading noise correlations in the diffusive metal but needs to be modified for higher moments [9]. Its main advantage is that it allows a unified treatment of the Johnson- and shot-noise regimes. When a strongly interacting system is driven out of thermal equilibrium, its current fluctuations cannot be neatly identified as Johnson noise or shot noise. Classification as Johnson noise is problematic, because out-of-equilibrium systems do not satisfy the usual fluctuation-dissipation relationalthough in certain cases there is an out-of-equilibrium analogue. Strong correlations induced by interactions lead to profound deviations from shot noise in the highfield regime, since charge is no longer carried in independent packets. One might anticipate, however, that a technique which gives a unified description of the Johnson- and shot-noise regimes may be applied to nonequilibrium, strongly interacting systems.

Here we apply the Boltzmann-Langevin approach to the study of strongly interacting, quantum critical bosons. In particular, we consider the superfluid to insulator transition of two-dimensional bosons with particle-hole symmetry. We use a Boltzmann-Langevin [7,8] approach within a $1 / N$ expansion [10] to analyze current noise near to this transition. The system is considered to be in thermal contact with a substrate. This, in conjunction with the internal scattering in the system, leads to a uniform, nonequilibrium, steady-state distribution, provided that the length of the sample is greater than the correlation length [5]. This situation is quite different from that considered elsewhere, where the only coupling to the outside world is through the leads. It permits the current-noise properties to be entirely determined by the internal scattering and so to be universal.

Field theory.-The critical region of the symmetric superfluid to Mott insulator transition phase diagram is described by a charged scalar field with a quartic interaction $[1,2]$ :

$$
\mathcal{H}=\int d^{d} x\left[\Pi^{\dagger} \Pi+\nabla \phi^{\dagger} \nabla \phi+m^{2} \phi^{\dagger} \phi+\lambda\left(\phi^{\dagger} \phi\right)^{2}\right],
$$

where $\phi$ is a complex scalar field and $\Pi$ is its conjugate momentum. These satisfy the usual commutation relations $[\phi(\mathbf{x}, t), \Pi(\mathbf{y}, t)]=i \delta(\mathbf{x}-\mathbf{y})$. We choose the bare interaction $\lambda$ to have its fixed point value $u^{*} \Lambda^{3-d}$, with momentum cutoff $\Lambda$ [1], although we will not need the precise, regularization-dependent, value. At the zerotemperature critical point, the renormalized mass is zero, which corresponds to a particular choice $m^{*}$ of the bare mass. The effects of applying an electric field $\mathbf{E}$ are included by minimally coupling to a vector potential; $\nabla \phi \rightarrow$ $D \phi=(\nabla+i e \mathbf{A} / \hbar) \phi$. We choose the gauge $\mathbf{A}=\mathbf{E} t$. This is equivalent to a contact-free measurement (for example, by placing the system on a cylinder and inducing an electromotive force on its surface by uniformly increasing the flux through it). We believe that our results carry over unchanged to the case with good contacts at the ends.

The normal modes of this Hamiltonian (in the absence of interaction) are charge-density fluctuations. These occur with positive and negative charges, corresponding to a decrease or increase in charge density of the superconductivity from the average. We determine the current fluctuations near to the critical point by considering a Boltzmann equation for the occupation of these modes. We use $a^{\dagger}$ and $a$ to represent the creation and annihilation, respectively, of positively charged density fluctuations and $b^{\dagger}$ and $b$ for negatively charged fluctuations.

The Boltzmann equation within a $1 / N$ expansion of the Hamiltonian (1) is given by [2,11]

$$
\left[\partial_{t}+(e \mathbf{E} / \hbar) \cdot \partial_{\mathbf{k}}\right] f_{\mathbf{k}}=\mathcal{S} r\left[f_{\mathbf{k}}\right]+e^{-\pi \hbar c \epsilon_{\mathbf{k}}^{2} / e E} \delta\left(\frac{\hbar \mathbf{k} \cdot \mathbf{E}}{e E^{2}}\right) .
$$

The first term on the right-hand side of this equation, $\mathcal{S} r[f]$, is the appropriate $1 / N$ expansion of the scattering integral $[2,5,10]$. The second term describes the production of particle-antiparticle pairs by the electric field through the Schwinger mechanism or Zener tunneling. This latter process is unimportant in the low-field limit but crucial in determining the correct high-field behavior [5].

$$
f(\mathbf{k}, t)=\int d \mathbf{q}\left\langle a_{\mathbf{k}+\mathbf{q} / 2}^{\dagger}(t) a_{\mathbf{k}-\mathbf{q} / 2}(t)\right\rangle
$$

denotes the distribution function, with a similar distribution $\tilde{f}(\mathbf{k}, t)$ for the negatively charged modes. Particle-hole symmetry implies the relation $f(\mathbf{k}, t)=\tilde{f}(-\mathbf{k}, t)$. We restrict our explicit consideration to the positively charged channel with final expressions including the negatively charged channel through appropriate factors of 2.

Before turning to a discussion of current fluctuations, we first review what is known about the solution of this Boltzmann equation in the high- and low-field limits. Near to the quantum critical point, all of the intrinsic energy scales of the system-and, in particular, those contained in the scattering integrals-renormalize to zero. The only remaining energy scales are those that are imposed externally. This is a recurring theme, which impacts upon both the form of the steady-state distribution 
functions and the fluctuations about them. In thermal equilibrium, the energy scale is provided by the temperature, and the distribution function is the usual Bose function of $\epsilon_{\mathrm{k}} / k_{B} T-\epsilon_{\mathrm{k}}$ being the energy of a normal mode with momentum k. In the high-field limit, the Schwinger source term [12] provides an energy scale $\propto \sqrt{e E / \hbar c}$. For later convenience, we make the suggestive identification of an effective temperature scale $T_{\text {eff }}(E)=\sqrt{\hbar c e E} / k_{B}\left(\pi^{2} / 4\right)$.

The low-field, thermal-equilibrium, distribution function is just that - a thermal Bose distribution. In Ref. [2], Damle and Sachdev calculated the leading corrections to this under the application of an electric field, in order to deduce the current - and, hence, the conductivity — in this low-field limit. They found that the leading corrections scaled as $\hbar c e E /\left(k_{B} T\right)^{2}$. On general grounds, corrections to higher order in field are expected to accumulate in powers of $\hbar c e E /\left(k_{B} T\right)^{2}$. Between the low- and high-field limits, therefore, the distribution function is a function of the ratios between $T, T_{\text {eff }}(E)$, and $\epsilon_{\mathbf{k}}$. This scaling of the distribution function, and the lack of energy scale in the scattering integral, is sufficient to determine our main results for the field and temperature dependence of current noise. Prefactors to scaling require detailed calculations which will be communicated elsewhere.

The Boltzmann-Langevin equation is an equation describing the stochastic evolution of fluctuations $\delta f_{\mathbf{q}}(\mathbf{r}, t)$ in the distribution function about its equilibrium or steady state. In the present case, it is given by

$$
\left[\partial_{t}+\mathbf{v} \cdot \partial_{\mathbf{r}}+(e \mathbf{E} / \hbar) \cdot \partial_{\mathbf{q}}\right] \delta f_{\mathbf{k}}=\gamma_{\mathbf{k}}[\mathbf{1}-\mathbf{M}]_{\mathbf{k q}} \delta f_{\mathbf{q}}+\eta_{\mathbf{q}},
$$

where we have used an Einstein convention with implied integration over the momentum label $\mathbf{q}$. The position and time labels of $\delta f_{\mathbf{k}}(\mathbf{r}, t)$ and $\eta_{\mathbf{q}}(\mathbf{r}, t)$ have been suppressed for compactness. The time and momentum derivatives on the left-hand side of Eq. (3) follow directly from the Boltzmann equation (2). The first term on the right-hand side is the linearized form of the scattering integral contained in Eq. (2); the kernel $\gamma_{\mathbf{k}}[\mathbf{1}-\mathbf{M}]_{\mathbf{k q}}$ describes $\operatorname{in}\left(\gamma_{\mathbf{k}} \mathbf{1}_{\mathbf{k q}}\right)$ - and out $\left(\gamma_{\mathbf{k}} \mathbf{M}_{\mathbf{k q}}\right)$-scattering processes. The second term is a stochastic term describing fluctuations in occupation number. It is determined by assuming that the scattering processes are independently Poisson distributed [13]. Under this assumption, the quadratic correlations of $\eta_{\mathbf{q}}(\mathbf{r}, t)$ are given by [14]

$$
\left\langle\eta_{\mathbf{q}}(\mathbf{r}, t) \eta_{\mathbf{q}^{\prime}}\left(\mathbf{r}^{\prime}, t^{\prime}\right)\right\rangle=2 \delta_{\mathbf{r}, \mathbf{r}^{\prime}} \delta_{t, t^{\prime}} \gamma_{\mathbf{q}^{\prime}} f_{\mathbf{q}}\left(1+f_{\mathbf{q}}\right)[\mathbf{1}-\mathbf{M}]_{\mathbf{q}^{\prime} \mathbf{q}} .
$$

The factor of 2 comes from the contributions of in- and outscattering processes.

Current fluctuations may be calculated using the Boltzmann-Langevin equation as follows: First, we determine the local fluctuations in occupation. In the limit of long times and long length scales, we may neglect the derivative terms on the left-hand side of Eq. (3). The fluctuation in the distribution function is then given by

$$
[1-M]_{\mathbf{k q}} \delta f_{\mathbf{q}}=\eta_{\mathbf{k}} / \gamma_{\mathbf{k}}
$$

which may be solved for $\delta f$. This result for $\delta f$ may be used to calculate the current fluctuations through

$$
\delta \mathbf{j}=\int d \mathbf{p} \mathbf{v}_{\mathbf{p}} \delta f_{\mathbf{q}},
$$

where $\mathbf{v}_{\mathbf{k}}=\partial_{\mathbf{k}} \epsilon_{\mathbf{k}}$. Using the noise correlations from Eq. (4), after some algebra, we obtain the following expression for the correlation of current fluctuations [14]:

$$
\left\langle\delta j_{\alpha}(\mathbf{r}, t) \delta j_{\beta}\left(\mathbf{r}^{\prime}, t^{\prime}\right)\right\rangle=\delta_{\alpha, \beta} \delta_{\mathbf{r}, \mathbf{r}^{\prime}} \delta_{t, t^{\prime}} \int d \mathbf{p} d \mathbf{q} \mathbf{v}_{\mathbf{p}} \cdot \mathbf{v}_{\mathbf{q}}[\mathbf{1}-\mathbf{M}]_{\mathbf{p q}}^{-1} f_{\mathbf{q}}\left(1+f_{\mathbf{q}}\right) / \gamma_{\mathbf{q}}
$$

This result should be compared with that for fermions [6], where the Bose enhancement factor $f+1$ is replaced by a Fermi factor $1-f$. The low frequency current noise $S_{j}$ can now be expressed as

$$
S_{j}=\int_{-\infty}^{\infty} d t d \mathbf{r}\langle\mathbf{j}(\mathbf{r}, t) \cdot \mathbf{j}(0,0)\rangle .
$$

Current fluctuations. - Our main results for the scaling of current fluctuations may be deduced by a scaling analysis of Eq. (7), using the scaling of the distribution function in its various limits, and the absence of energy scale in the scattering integrals at the critical point.

In the low-field limit, the distribution is a function of $\epsilon_{\mathbf{q}} / T$. Rescaling all of the momentum integrals in Eq. (7) by $T$ gives $S_{j}^{T} \propto T$. Note the dimensions of $\left[\gamma_{\mathbf{q}}\right]=[\omega]=$ $[c \mathbf{q}]$ and $[\mathbf{1}]=[d \mathbf{q}]^{-1}$. In the high-field limit, the distribution is a function of $\epsilon_{\mathbf{q}} / T_{\text {eff }}(E)$, and a similar scaling analysis of Eq. (7) gives $S_{j}^{E} \propto T_{\text {eff }}(E) \propto \sqrt{E}$. Finally, since the corrections to the distribution function away from thermal equilibrium may be expanded in powers of $T_{\text {eff }}(E) / T$, the current noise is expected to be given by a universal scaling function

$$
S_{j}=T \Phi\left[T_{\text {eff }}(E) / T\right],
$$

with low-field limit $\Phi[x \rightarrow 0]=$ const and high-field limit $\Phi[x \rightarrow \infty]=$ const $\times x$. The determination of the constant prefactors in the high- and low-field limits of this scaling function requires detailed calculation. The functions $\gamma_{\mathbf{q}}$ and $M_{\mathbf{k}, \mathbf{q}}$ determining the scattering integrals may be deduced within a $1 / N$ expansion both in thermal equilibrium $[2,10]$ and in the high-field limit [5]. The integrals in Eq. (7) may be carried out using these combined with the explicit forms of the distribution functions. In the low-field limit, we obtain $\Phi[x \rightarrow 0]=4 \sigma_{T}$, where $\sigma_{T}=$ $0.2154 e^{2} / h$ is the universal conductance found in thermal equilibrium [1,2]. This is in accord with the equilibrium 
fluctuation-dissipation relation - as, of course, it must be. In the high-field limit, we obtain $\Phi[x \rightarrow \infty]=4 \sigma_{E} x$, where $\sigma_{E}=(N \pi / 8) e^{2} / h$ is the large-field, out-ofequilibrium conductivity [5]. The reasons for our rather curious choice of numerical prefactor in the definition of $T_{\text {eff }}(E)$ can now be seen. With the present choice, the highfield current noise may be written in the form $S_{j}=$ $4 \sigma_{E} T_{\text {eff }}(E)$, reminiscent of the thermal-equilibrium form demanded by the fluctuation-dissipation relation. Our choice of $T_{\text {eff }}(E)$ allows an identification of an out-ofequilibrium analogue of the fluctuation-dissipation relation.

Discussion. - As we have seen above, the scaling of current noise with field and temperature is ultimately due to the vanishing of internal energy scales at the quantum critical point. The only remaining energy scales are those imposed externally through the temperature of the environment or by applied fields. Although the high-field noise cannot be unambiguously identified as either Johnson noise or shot noise, it is nevertheless revealing to make a comparison with these limits. We have already anticipated the comparison with Johnson noise by our identification of an effective temperature $T_{\text {eff }}(E)$. From this perspective, our calculation is a microscopic derivation of the out-ofequilibrium analogue of the fluctuation-dissipation relation. Comparison with shot noise is more difficult. The large-field current noise $\left(S_{j} \propto \sqrt{E}\right)$ is dramatically reduced compared with the linear $\mathbf{E}$-field dependence of shot noise expected for uncorrelated charge carriers. It is tempting to ascribe this to an effective charge of carriers near to the quantum critical point proportional to $1 / \sqrt{E}$. This effective charge shows a dramatic divergence as one approaches the quantum critical point, reflecting the strong correlations of the system. Whether this picture can be carried through requires an analysis of higher order statistics of the current noise [9].

We have concentrated in this Letter upon the theoretical analysis of current fluctuations. We have shown how they may reveal aspects of quantum critical scaling that are complementary to those revealed by response functions in the high-field limit. How do these effects impact upon experiment? There are a variety of systems where one might expect our analysis to apply and where, consequently, measurement of current noise is expected to be a useful tool. Examples include the SI transition in thin films such as MoGe and the SI transition at the underdoped side of the high-temperature superconductor phase diagram. These results may also apply, mutatis mutandis, to fractional quantum Hall plateau transitions. A rough estimate of the magnitude of this effect in a physical setting such as the SI transition in MoGe thin films [15] may be obtained by neglecting the effect of disorder and taking $E=$ $0.5 \mathrm{~V} / \mathrm{m}$ and $c \sim v_{F}=10^{6} \mathrm{~m} / \mathrm{s}$. We obtain $T_{\text {eff }} \sim$ $400 \mathrm{mK}$, which is an order of magnitude larger than experimentally accessible temperatures, meaning that the current noise is enhanced over its thermal value.
In conclusion, we have considered the current noise at the two-dimensional, $z=1$ superconductor to insulator quantum critical point. We find that this noise follows a universal scaling function $S_{j}=T \Phi\left[T_{\text {eff }}(E) / T\right]$, with $T_{\text {eff }}=\sqrt{\hbar c e} E \pi^{2} / 4 k_{B}$. This scaling function recovers Johnson noise in thermal equilibrium and crosses over to an unusual $\sqrt{E}$-dependent nonlinear shot noise or nonequilibrium Johnson noise at high fields. This is a particular case of a more general $E^{z /(1+z)}$ scaling expected for highfield current noise. In this way, current noise may reveal the universal nonlinear scaling exponents predicted near to quantum phase transitions.

This work was completed with the support of the Royal Society, NSF No. DMR-0238760, the LDRD program of LBNL under DOE Grant No. DE-AC02-05CH11231, and the A. P. Sloan Foundation (A. V.). We thank D. Gutman and N. Mason for discussions.

[1] S. Sachdev, Quantum Phase Transitions (Cambridge University Press, Cambridge, England, 1999); see also S. L. Sondhi et al., Rev. Mod. Phys. 69, 315 (1997).

[2] K. Damle and S. Sachdev, Phys. Rev. B 56, 8714 (1997).

[3] M.-C. Cha, M. P. A. Fisher, S. M. Girvin, Mats Wallin, and A. P. Young, Phys. Rev. B 44, 6883 (1991).

[4] D. Dalidovich and P. Phillips, Phys. Rev. Lett. 93, 027004 (2004).

[5] A. G. Green and S. L. Sondhi, Phys. Rev. Lett. 95, 267001 (2005).

[6] See, for example, Eq. (8) of K. E. Nagaev, Phys. Rev. B 57, 4628 (1998) or Eq. (9) of K. E. Nagaev, Phys. Rev. B 66, 075334 (2002).

[7] M. Bixon and R. Zwanzig, Phys. Rev. 187, 267 (1969).

[8] Sh. M. Kogan and A. Ya. Shulman, Zh. Eksp. Teor. Fiz. 56, 862 (1969) [Sov. Phys. JETP 29, 467 (1969)].

[9] D. B. Gutman, Yuval Gefen, and A. D. Mirlin, cond-mat/ 0210076; K. E. Nagaev, Phys. Rev. B 66, 075334 (2002).

[10] S. Sachdev, Phys. Rev. B 57, 7157 (1998).

[11] We use the shorthand notation $d \mathbf{q}=d^{2} q /(2 \pi)^{2}$ for the momentum-space integration measure.

[12] An alternative (although essentially equivalent) way to deduce this latter energy scale is through a dimensional analysis of the Hamiltonian (1); in carrying out this analysis, one finds $[\omega]=[e E t / \hbar]$, which after suitable rearrangement gives an energy scale $\sqrt{e E / \hbar c}$.

[13] This is valid provided that the scattering can be expanded perturbatively. Each term in the perturbative expansion describes a separate, independent process that contributes to fluctuations. In strongly correlated systems, such as the one that we consider here, this expansion is controlled by a $1 / N$ or $\epsilon$ expansion.

[14] In fact, there is a subtlety here. The particle-hole scattering processes induce correlations between noise in the particle and hole channels. Writing the noise correlators as in Eq. (4) incorporates these effects in such a way that we may treat particle and hole scattering as if they were independent, obtaining the correct result.

[15] N. Mason and A. Kapitulnik, Phys. Rev. Lett. 82, 5341 (1999). 